des Fusses, welehe allein durch die Schwere bedingt, wird bei Nichtgebrauch des Fusses, wenn Hüft- und Kniegelenk infolge schwerer Coxitis in Flexion fixirt sind durch tendo-, resp. myoxene Contractur. Bei Kranken, die olne ärztliche Behandlung in frühem Alter die tuberkulöse Entzündung des Hüft- und Kniegelenlss überstanden haben, entstelıt, wie bekannt, secundär durch die rom Kranken innegehaltene Stellung des Beines eine Contractur in den betreffenden Gelenkenl. Nach Ablauf der Erscheinungen müssen sie sich mit Hülfe voll Krïcken weiter bewegen. Der Oberschenkel ist flectirt, der Unter'schenkel auch, und der sonst resunde Fuss liängt unbenutzt, der Scliwere folgend, herab. Es sind für ihn dieselben Bedingungen gescliaffen, wie für den paralytischen Fuss. Weshalb nuı beim schlaffen Herabhängen des sonst normalen Fusses eill Pes varo-equinus entstelit, erkläre ich mir aus dem anatomischen Bau des oberen Sprungrelenks und dein Gesetz der Schwere. Denke ich mir den Fuss als Ganzes, so ist derselbe gewissermaassen an den Bandmassen aufgehängt, die das obere Sprunggelenk umgebell, welln die Muskelwirkung ausgeschaltet ist. Die Fussspitze sinkt nach unten, weil der vordere Hebelarm (der Fuss z. B. als Stab gedachț) grösser ist. Soll eiı Fuss - und dieses sielıt man am kesten an nornıalen knöclıernen Präparaten - horizontal liegen, so muss ich illu ungefähr in dem Winkel zwischen Naviculare, Cuboid und Cuneiforme III suspendiren. Dass bei dem Herabsinken der Fussspitze zugleich eine Drehung des Fusses um seine Achse liacl aussen (Supination) erfolgt, liegt ill der Gestalt des oberen Sprunggelenks. Es ist dasselbe ein Theil einer rechts gedrehten Schraube, der äussere Rand der Talusrolle liegt lıölıer.

Es giebt deminacl keine reille Flexions- und Extensionsbewegulıg im oberen Sprulggelenk, es silld Sclıraubenbewegungen. Man denke sich den 'Talus als eine Schraube, die man in die Tibia schraubt. Will icll die Schiaube. d. h. den Talus, herausschrauben, so müsste ich den Fuss aus der ilormalen Stellung nach innen drehen und supinireu. Selir leicht kann man an eigenen Fusse selıen, dass die sogenaunte Dorsal-, resp. Volarflexion gar keine Flexionsbewegung ist, sondern die grosse Zele z. B. beschreibt in der Luft die Bahı einer Schraubenwindung, alles bei fixirtem Unterschenkel. Wer darauf bisher nicht geachtet liat, wird die Schraubenbewegung zu Begiun der Volarflexion z. B. kaum sehen; sobald der Fuss inelir volar flectirt wird, tritt die Schraubenbewegung deutliclı zu Táge.

Dies sind nach nleiner Ansicht die Gründe, weshalb beim einfachen Herabhäugen des Fusses und Inactivität der Muskeln nur ein Pes varo-equinus eintritt. Dies sind zwei Bedingungen, die sowohl bein paralytischen Fuss als auch bei Contractur des Hüft- und Kniegelenks zutreffen, indem in letzterem Falle eine Thätigkeit der Fussmuskelı nutzlos, ja unmöglich wird, denn dio zum F'uss gehenden Extensoren 11 üssten, um den Fuss in normaler, zuın Unterschenkel rechtwiukliger Stellung zu erhalten, daueı'nd colitraliirt bleiben. Vielleicht kollmt nocli linzu, dass mit der Zeit die combinirte Fussbewegung verlernt wird, so dass der Fuss schliesslich schlaff, wie der paralytische, leerabliängt.

Hier will ich eine Besclireibung der Muskulatul des Fusses geben, wie sie siclı beim postfötal erworbenen Klumpfuss findet. Die Lageveränderungen an den Sehnen und Muskeln des Fusses sind Secundärerscheinungen, bedingt durclı die veränderte Lago und Gestalt der Fussknochen. Im allgemeinen ist die Muskulatur atrophisel, zum Theil fettig degenerirt.

Beginnen wir mit den Extensoren. Der Extensor digitorum c o m m lin is longus sendet nornal seine Endsehne, resp. das schon gesonderte Sehmenbiindel durch das lateral gelegene Fach rles Ligamentum cruciatum, diese geht dann gerade weiter auf dem Fussrücken, um sich fächerförniig an die zweite bis fünfte Zehe zu vertheilen. Beim Pes varo-equinus wird die Sehne durch das starke Hervorspringen der Talocruralgelenkfläche gezwungen, eine s-förmige Biegung zu machen. Sie liegt zunäclist lateral, der vorderen lateralen Ecke der Talocruralgelenkfläche an, dann um diese Ecke medialwärts biegend, verläuft sie ïber den Taluskopf ill gewölnnliclier' Weise zu den Zehen.

Die Sehne des Extensol hallucis longus kommt gleichfalls durch die veränderte Stellung des vorspringenden Talus in Berülırung mit der Talocruralgelenkfläche. Die Sehıe liegrt über dem medialen Drittel der betreffenden Gelenkfläclıe, dicht auf den Knochen, nur durch die weit nach vorn ausgezogene Gelenkkapsel

\section{Beiträge zur Anatomie des Pes varo-equinus.}

Von Dr. E. Funke, Königsberg i. Pr.

Die Ursache des Klumpfusses ist, wie wir wissen. äusserst verschieden. Es giebt myogene, desmogene, arthrogene und paralytische Klumpfüsse. Ich möchte darauf aufmerksam machen, dass es auch Inactivitätsklumpfüsse giebt. Es ist dieses die Stellung von der Gelenlufläche des Talus getrennt. Die Sehne ist gewissermaassen wie eine Saite ïber den Steg der Violine, so über den nach vorn ragenden freien Theil der oberen Gelenkfläche des Talus gespanit.

Durch das am nieisten median gelegene Fach verläuft normal die Sehne des M. tibialis anticus an die mediale Seite des Gelenkes zwischen Cuboideus I und Metatarsus I und endigt, in die Gelenkkapsel ausstrahlend, an einell Höcker der Basis des ersten 
Metacarpus und einer lacette und der vorderen, medialen und unteren Kante des ersten Keilbeins. Dieser Ansatz des Tibialis anticus ist etwas flacher gegen den Knochen gerichtet beim Pes varo-equinus, d. h. die Selne ist gewissermaassen straffer angezogen. An der Talusrolle liegt die Selne medial von der Sehne des Extensor lallucis longus, diclit an dem vorderen Theile der medialen Kante der oberen Gelenkfläche des Talus.

Die Selıne des Peroneus longus ändert mit den Knochen ilnre Lage, da sie in festen Scheiden liegt. Während die Selne bei lorizontal liegendem Fusse an der lateralen Seite des Calcanens von hinten oben nach voln unten, also schräg verläuft, ändert sich dieser Verlauf durelı Senken der Fussspitze in einen senkrechten. Dann liegt weiterhin die Sehne beim Pes varo-equinus am Cuboid schräg, in der Fusssohle selbst horizontal. An der Vorderfläche der Cuboidrolle vorbei verläuft die Sehne durch Bindegewebe an die Insertionsstelle des Abductor digiti V. in der Nähe der medialen, volaren Ecke der Basis metatarsi V. angeheftet zur Fusssohle. Ich möchte bemerken, dass die Peroneussehne sowohl hier, als in jedem anderen Falle nicht ïber die Rolle des Cuboid geht, sondern an der Vorderfläche der Rolle liegt. Das zweite (tiefere) Blatt des Ligamentum calcaneo-cuboideum reicht bis an den freien Rand der Rolle (Fig. 59). In der Planta theilt sich die Peroneussehne in drei $Z$ weige. Der mittlere stellt die direkte Fortsetzung der Sehne dar und setzt sich an die grosse laterale, dreieckige Tuberositas der Basis metatarsi I. (nicht an die untere). Der hinter'e Schenkel wendet sich in sanftem Bogen nach linten zur lateralen, vorderen und unteren $T$. Tuberositas des ersten Keilbeins. Fig. 59 .

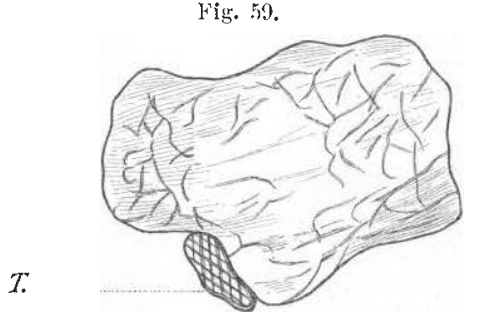

Der vordere strangartige Schenkel geht scluräg nach vorn in die Tiefe zum Musenlus interossens. Die Selme des Peroneus longus theilt die Planta pedis in zwei Gruben (nach Entfernung von Haut und Unterliautgewebe). Die vordere bildet ein in der Planta schläg: von hinten lateral nach voln medial verlanfendes Oval, die lintere wird begrenzt vom Liganentum calcaneo-cuboirleun, der Selne des Tibialis posticus und der Peroneus longus-Sehne. Vom Peroneus brevis ist niellts besonderes zu sagen, auch seine Endsehne verläuft, entsprechend der Senkung der Fussspitze, meln' senkrecht.

Die Selne des Flexor digitorum communis tritt zunächst in die Furche zwischen Sustentaculum tali und dem Hinterrande des Malleolus internus, da wo die Rinue des Musculus tibialis posticus an der Tibia endigt, sie liegt damn 5 mm weit dem Ligannentum calcaneotibiale auf, dann an der linteren Hälfte des Processus medialis posticus tali, wendet sich schräg am vorderen Theile des Sustentaculum vorbei zur Planta. Dort spaltet sich die Sehne in zwei Theile. Der eine Theil geht neben der ersten normalen zum Digitus II. Der Tibialis posticus konmt mit seiner Selne vom Sulcus an der Hinterseite der Tibia herab an den Hinterrand des Malleolus internus, in einer tiefen Rinne liegend. Die Sehne setzt sich zunäclist sehr fest und breit an das Ligamentum calcaneonaviculare, von hier aus verbreitert sie sich fächerförmig; die direkt fortgesetzten Fasern gehen unter dem Naviculare weg, heften sich an die hintere, untere Tuberositas des ersten Keibeins.

Von hier gehen Fasern weiter zur unteren medialen Tuberositas metatarsi I. Die übrigen Fasern ziehen fä̈cherförmig' unter dem lateralen Theil des Naviuulare und dem medialen des Cuboideum zum Ligamentum calcaneo-cuboideum, resp. zu den Ossa cuneiformia I und II und der Selmenscheide des Peronens longus. Der Abductor hallucis liegt medial um das Sustentaculum tali verlaufend. Er entspringt normal auch vom medialen Rande des Naviculare, da wo der Tibialis posticus ansetzt. Fermer von den Insertionsstellen des Tibialis anticus, vom Ligamentum laciniatum und der Tuberositas calcanei. Der Abrluctor hallucis war stark entwickelt. Anı Abductor digiti V. sowie den Interossei nichts besonderes. Der Muscullus extensor lallucis brevis inserirt mit einer Reihe von Fasern an lateraler Seite des Talushalses und der Naviculares. Wemn Hoffa (Lehrbuch der orthopädischen Chirurgie) schreibt: „Die Sehnenrinne des Musculus peroneus longus findet sich statt am Talus am Processus anterior calcanei", so kann ich nur sagen, dass sie sicll niemals am Talus findet, sondern dass das Retinaculum peroneorum, resp. der Processus trochloaris sich stets an der lateralen Fläche des Calcaneus findet.

Der Fuss wird in seiner Varusstellung hauptsächlich durch die verkiirzten Ligamentum talo-tibiale und calcaneo-tibiale internum gehalten, ebenso ist das Ligamentum laciniatum internum stark verkïrzt, nur $3 \mathrm{~cm}$ lang (von Pes varo-equinus des Erwachsenen). Die obere T'alusgelenkfläche lag' ca. $21 / 2 \mathrm{~cm}$ breit und $1 \frac{1}{2} \mathrm{~cm}$ in sagittaler Richtung frei. 